\title{
ANÁLISIS DE LAS NORMAS JURÍDICAS - ELABORADAS PARA LA IMPLEMENTACIÓN DE LA SILLA VACÍA COMO MECANISMO DE PARTICIPACIÓN CIUDADANA EN LAS SESIONES DE LOS CONCEJOS MUNICIPALES DEL ECUADOR
}

\author{
ANALYSIS OF THE LEGAL STANDARDS - ELABORATED FOR THE \\ IMPLEMENTATION OF THE EMPTY CHAIR AS A MECHANISM OF CITIZEN \\ PARTICIPATION IN THE SESSIONS OF THE MUNICIPAL COUNCILS OF
}

ECUADOR

\section{RAFAEL COMPTE GUERRERO}

Universidad Católica de Santiago de Guayaquil.rafael.compte@cu.ucsg.edu.ec

RESUMEN

En el presente artículo, se determina la eficiencia de las normas jurídicas elaboradas y aprobadas por los municipios en el marco de su facultad legislativa, a efectos de normar la implementación de la Silla Vacía. Este mecanismo, tiene el objetivo de dar cumplimiento al mandato constitucional y legal, de posibilitar la participación ciudadana. Se realizó un estudio de las Ordenanzas de veintiséis municipios del Ecuador. Fueron establecidas cinco categorías de análisis, a efectos de determinar si las mismas cumplían con la norma constitucional y legal que posibilita el acceso de la ciudadanía a esta forma de participación. Se determinó que las ordenanzas analizadas no posibilitan su implementación, pues incumplen la Constitución y la Ley, por lo que se recomienda una propuesta de Ordenanza que contemple lo previsto en la normativa existente; esto es, la Constitución de la República del Ecuador, la Ley Orgánica de Participación Ciudadana y el Código Orgánico de Organización Territorial, Autonomía y Descentralización (COOTAD).

PALABRAS CLAVE: Constitución, participación ciudadana, municipios, silla vacía.
The efficiency of the Ordinance, legal standard developed and approved by the municipalities within the framework of its legislative power, in order to regulate implementation of the Empty Chair, to comply with the constitutional and legal mandate to enable citizen participation is determined. a study of the Ordinances of twenty six municipalities of Ecuador, on the basis of establishing five categories of analysis, in order to determine whether they complied with the constitutional and legal standard that enables access of citizens to this form of participation was made. It was determined that the analyzed Ordinances not allow its implementation because violate the Constitution and the law, so that a proposed ordinance that if contemplates the provisions of the existing legislation is recommended; that is, the Constitution of the Republic of Ecuador, Law organic Citizen Participation and the organic Code of Territorial Organization, Autonomy and Decentralization (COOTAD).

KEYWORDS: Constitution, citizen participation, municipality, empty chair. 
INTRODUCCIÓN

Los Gobiernos Autónomos Descentralizados (GAD) están llamados a promover el desarrollo sustentable de su circunscripción territorial para garantizar la realización del Régimen del Buen Vivir a través de la implementación de políticas públicas, en el marco de sus competencias constitucionales y legales. Para ello, entre otras consideraciones, deben implementar un sistema de participación ciudadana para el ejercicio de los derechos y la gestión democrática de su acción (COOTAD, 2010).

En la Constitución ecuatoriana se ha estructurado un sistema de participación ciudadana inédito en el Ecuador contemporáneo, tal como se evidencia, principalmente, en los artículos 204, 207, y 208. En concordancia con el marco de la creación de la Función de Transparencia y Control, se incluyó la figura de la "Silla Vacía" en los GAD. Esto incorpora, la posibilidad de que un ciudadano ejerza, con voz y voto, la representación ciudadana en las sesiones de su órgano legislativo.

La idea es que, además de las "sillas" ya ocupadas por los concejales (representantes del voto popular), haya una "silla" que esté "vacía", pues en ésta debería estar alguien de la ciudadanía, actora y receptora de las decisiones de los gobernantes y que debe participar en lo que allí se decida, pues atañe directamente a sus intereses individuales y/o comunitarios. La normativa existente (Constitución de la República del Ecuador, la Ley Orgánica de Participación Ciudadana y el COOTAD), establece que dicha participación se concretará, cuando en una sesión determinada se vaya a discutir un tema en el cual sea pertinente la representación que determinado ciudadano ejerza.

El novedoso mecanismo constituye una puesta en práctica efectiva de democracia participativa. El Ecuador es un país donde ha existido una democracia representativa y donde las autoridades de gobierno han sido electas por votación (universal y directa) y representan la voluntad ciudadana. Sin embargo, constituye un debate muy interesante el plantearse si este mecanismo de la Silla Vacía es una mera delegación ciudadana o puede convertirse en una representación para que su accionar sea una verdadera participación efectiva en la toma de decisiones del nivel de gobierno que corresponda. Los resultados podrán vislumbrarse, con el transcurso del tiempo, conforme se vaya aplicando.
En efecto, la participación ciudadana es un derecho. Los ciudadanos deben ser parte de la toma de decisiones, de la planificación y la gestión de los asuntos públicos, así como del control popular de las instituciones del Estado. A la vez, la participación de la población en la formulación de políticas públicas es un elemento fundamental para la realización del Régimen del "Buen Vivir".

En este sentido, es una prioridad recoger la voz de aquellos que en escasas ocasiones han tenido la oportunidad de expresar sus sueños, aspiraciones y cosmovisiones; pero también es un deber establecer canales de diálogo con la ciudadanía organizada a fin de fortalecer la democracia (SENPLADES, 2009).

Sin embargo, es importante plantearse qué características debe tener el mecanismo de la Silla Vacía para que se constituya realmente en una instancia de participación ciudadana, como sostiene Pachano (2011). El mismo, considera que es necesario ofrecer condiciones de igualdad para todos los grupos y en general para todos los ciudadanos interesados en un tema determinado. La Silla Vacía debe ser entendida como un procedimiento estrictamente democrático, del que pueden hacer uso todos los ciudadanos que lo deseen, sin impedimentos de ninguna naturaleza. Adicionalmente, deberán estar claramente definidas las atribuciones de las personas que hagan uso de este recurso, especialmente en lo que se refiere al alcance de su derecho.

Idealmente, la Silla Vacía debería ser un espacio de expresión de las diversas posiciones que existen en torno a un tema determinado, de manera que no puede ser adjudicada a una sola persona u organización. Además, la Silla Vacía debe ser entendida como un espacio en que los diversos sectores de la sociedad exponen sus respectivas posiciones acerca de un tema determinado, de modo que no debe ser una modalidad de definición de políticas. En estricto sentido, es una instancia de lobbying o de cabildeo, mas no de mera comunicación de lo ya decidido. Para el ejercicio de esta participación se organizarán audiencias públicas, veedurías, asambleas, cabildos populares, consejos consultivos, observatorios y las demás instancias que promueva la ciudadanía (Acosta, 2011).

Ahora bien, cuando hablamos de participación ciudadana, debemos tener claro de qué tipo de participación estamos hablando. Cuando se refiere a tomar parte en los asuntos públicos, implica una mayor distribución del 
poder respecto de ellos ("tomar parte" del poder) y se convierte en un método de profundización democrática. Así entendida, la participación no se alcanza, ni mucho menos se agota, en la consecución de alguno de sus escalones: información, consulta, toma de decisiones o gestión colegiada. Cada uno de estos niveles forma parte de un continuum y a la vez requisitos para que la participación pueda desarrollarse. En función de los objetivos que la participación persiga, del nivel que se logre y del papel que desempeñen los ciudadanos, podemos hablar de participación plena, participación incompleta o incluso de pseudo participación (Alberich y Espadas, 2011).

También puede entenderse como una recuperación del "espacio público", pues estos posibilitan el encuentro y la interacción entre personas, contribuyendo a la tolerancia entre grupos sociales diversos y a la construcción del sentido de vida colectiva (Burneo, 2011).

En ese sentido, lo ideal es que exista una democracia participativa que se diferencia de la representativa, en tanto es una democracia esencialmente activa. Es decir, es una democracia que requiere para su propia supervivencia de la acción de sus sujetos protagonistas, que son los ciudadanos (Ramírez, 2009).

Es importante señalar, que no existe en el Ecuador una investigación que dé cuenta de los resultados obtenidos con la implementación de la Silla Vacía en los GAD. Son escasos también los estudios que existen sobre temas afines. Uno de ellos es el realizado sobre el fortalecimiento de la organización barrial en Loja, en el que se cuestionan, ¿Cómo fortalecer la organización barrial lojana en las actuales circunstancias políticas? Concluyen, entre otras consideraciones, que debe ser a partir de la potenciación de los liderazgos naturales en los barrios.

Este proceso es fundamental en el actual esquema democrático ecuatoriano, después de haber entrado en vigor el nuevo Código Orgánico de Ordenamiento Territorial, Autonomía y Descentralización, que otorga estatus de gobierno descentralizado autónomo hasta el nivel de junta parroquial, ya que es muy importante contar con liderazgos populares que gestionen desde abajo (Romero y Loja, 2011).

Con estas consideraciones, el objetivo de la investigación (que sirvió de base a este artículo) fue determinar si los municipios seleccionados, han aprobado las ordenanzas que posibiliten la implementación de este mecanismo de participación ciudadana.
Otro eje de la investigación determinó, si el mecanismo es utilizado por la ciudadanía, con qué frecuencia, en qué temas, por cuáles actores, cuánto ha influido su participación, y si la comunidad se ha beneficiado o no.

\section{METODOLOGÍA}

Para la realización de la investigación, se seleccionaron 26 cantones de los 221 que exiten en el país:

Guayaquil con una población de 2.350.915 habitantes; Quito con 2.239.191; Cuenca con 505.585; Santo Domingo de los Tsáchilas con 368.013; Ambato con 329.856; Portoviejo con 280.029; Machala con 245.972; Riobamba con 225.741; Loja con 214.855; Ibarra con 181.175; Latacunga con 170.489; Milagro con 166.634; Babahoyo con 153.776; Santa Elena con 144.076; Chone con 126.494; Otavalo con 104.874; Lago Agrio con 91.744; Orellana con 72.795; Azogues con 70.064; Pastaza con 62.016; Sucre con 57.159; El Guabo con 50.009; Montalvo con 24.164; Patate con 13.497; Chunchi con 12.686; y, Palora con 6.936.

La muestra escogida, contiene la diversidad de nuestra realidad social y política, desde Guayaquil con sus casi tres millones de habitantes, hasta Palora de la provincia de Morona Santiago, con apenas ocho mil habitantes. Cabe señalar que el total de la población de estos 26 cantones asciende a 8’268.742 habitantes, lo que representa el $57.09 \%$ del total de la población ecuatoriana (14`483.499 habitantes) según lo establece el Censo de Población y Vivienda del 2.010 realizado por el Instituto Nacional de Estadística y Censos (INEC, 2010).

La investigación fue descriptiva. Se inició determinando cuáles de los municipios seleccionados, cuentan con una ordenanza aprobada por su Concejo Cantonal. Luego, contando con las categorías de análisis que se establecieron, determinar si dichas ordenanzas se encuentran estructuradas de tal forma, que no solo posibiliten real y efectivamente la participación ciudadana, sino que contemplan en su contenido, lo establecido en la norma constitucional y leyes pertinentes.

En efecto, resulta necesario determinar si las normas jurídicas de la Ordenanza, cumplen con lo dispuesto en la norma constitucional y legal. Para ello se consideró analizarlos a la luz de cinco categorías de análisis, que den cuenta de consideraciones fundamentales que toda Ordenanza debe contemplar: 
a. Si las sesiones eran efectivamente públicas;

b. Si la representación ciudadana no tenía limitaciones;

c. Que no se limiten los temas a ser tratados y discutidos;

d. Que la ciudadanía al intervenir y debatir no encuentre restricciones; $y$

e. Que se establezca la sanción que tendría la autoridad que no posibilite la participación ciudadana.

TÉCNICAS Y MODELOS DE ANÁLISIS DE DATOS

Se utilizó el análisis de contenido como método cualitativo, que incluyó la codificación, categorización y triangulación de la información obtenida, a través de las categorías de análisis escogidas según se muestra en la tabla 1.

\section{TABLA 1: CATEGORÍAS DE ANÁLISIS}

Carácter público de las sesiones de los Concejos Cantonales
Que quién ocupa la Silla Vacía sea un representante de la ciudadanía sin
limitaciones
Que no haya limitación ni restricción de los temas a tratarse
Que la participación ciudadana en el debate esté garantizada, sin
limitaciones no coerciones
Que se prevea la sanción que le puede acarrear a la autoridad pública que
incumpla el mandato constitucional y legal

Se establecieron cuatro posibilidades en base a los resultados encontrados: A) Cumplimiento Total; B) Cumplimiento Parcial; C) Incumplimiento; y D) Inconstitucionalidad e ilegalidad.

Cada posibilidad, se ha aplicado a cada una de las cinco categorías de análisis propuestas.

RESULTADOS

Se evidenció la forma en que los municipios seleccionados cumplen o no, con las categorías de análisis propuestas.

Carácter público de las sesiones de los Concejos Cantonales, a través de una convocatoria que llegue a toda la ciudadanía

Los municipios de Guayaquil, Cuenca, Loja, Lago Agrio, Azogues, Montalvo y Sucre, cumplen de manera satisfactoria con esta categoría, inclusive incorporando mecanismos como difusión por la página web oficial del Municipio, la radio municipal, entre otros.

Los cantones Babahoyo, Chone, Orellana, Pastaza, Chunchi y El Guabo, cumplen solamente de manera aceptable; pues no son precisos y específicos en los mecanismos de difusión.
Los municipios de Ambato, Portoviejo, Machala, Ibarra, Latacunga, Milagro y Palora, no cumplen de manera satisfactoria pues no prevén una difusión amplia de la Sesión.

Que quién ocupe la Silla Vacía sea un representante de la ciudadanía sin limitaciones

Los municipios de Cuenca, Ibarra y Chunchi permiten una participación ciudadana tanto de manera individual como colectiva, sin requisitos o limitaciones.

Los cantones de Ambato, Machala, Loja, Latacunga, Babahoyo, Chone, Lago Agrio, Orellana, Azogues, Montalvo, Sucre y Palora, permiten tanto la participación individual como colectiva, pero con requisitos que el ciudadano debe cumplir.

Los municipios de Guayaquil, Portoviejo, Milagro, Pastaza y El Cuabo, solo permiten la participación si el ciudadano pertenece a una organización y cuenta con su autorización.

Que no haya limitación, ni restricción de los temas a tratarse

Los municipios de Guayaquil, Cuenca, Milagro, Ibarra, Chunchi, Ambato, Machala, Loja, Latacunga, Chone, Lago Agrio, Orellana, Montalvo, Pastaza y Sucre no evidencian limitación, ni restricción de los temas a tratarse.

Los municipios de Portoviejo, Babahoyo, Azogues, El Guabo y Palora, establecen varios temas donde podrá existir participación ciudadana a través del mecanismo de la Silla Vacía.

Que la participación ciudadana en el debate esté garantizada, sin limitaciones, ni coerciones

Los municipios de Guayaquil, Ambato, Loja, Ibarra, Babahoyo, Chone, Orellana, Azogues, Montalvo, Sucre, El Guabo y Palora se acogen a lo dispuesto en la Ley Orgánica de Participación Ciudadana. Esto significa que en caso de posturas ciudadanas diferentes, habrá necesidad de buscar consenso y de no conseguirlo habrá sorteo. En ese caso la participación será solo con voz.

Los municipios de Machala, Portoviejo, Milagro, Lago Agrio y Pastaza, no establecen de manera clara y precisa que hacer en caso de la existencia de dos posturas ciudadanas diferentes.

Los de Cuenca, Latacunga y Chunchi, establecen serias limitaciones a la participación ciudadana, coartando la posibilidad de expresar su opinión a través del voto.

Que se prevea la sanción que le puede acarrear a la autoridad pública que incumpla el mandato constitucional y legal 
Solo los municipios de Portoviejo, Loja, Chone y Azogues señalan cuáles serían las sanciones que tendría la autoridad que no posibilitara la participación ciudadana. El resto de municipios no señalan nada al respecto.

Del análisis de las ordenanzas analizadas se puede establecer lo siguiente:

Seis $(23,08 \%)$ de las ciudades escogidas para la investigación, no han aprobado ordenanza alguna; para normar el mecanismo de participación ciudadana denominado Silla Vacía, incumpliendo con esto el mandato constitucional y legal (lo que resulta grave y preocupante).

De los veinte $(76,92 \%)$ cantones que sí han aprobado la Ordenanza correspondiente, trece $(65 \%)$ de ellos lo han hecho para disponer sobre la participación ciudadana o el funcionamiento de las sesiones del Concejo Cantonal; y siete (35\%) para la Silla Vacía. Un Concejo Cantonal (Cuenca) normó el mecanismo de la Silla Vacía, aún antes de la vigencia de las leyes secundarias para el efecto (la Ley Orgánica de Participación Ciudadana y el COOTAD).

Estas leyes LOPEC y COOTAD fueron aprobadas en agosto de 2009 y octubre de 2010 res- $^{-}$ pectivamente. Desde entonces, los municipios tienen la obligación de dictar la Ordenanza que regula los mecanismos de participación ciudadana, concretamente el de la Silla Vacía, objeto de esta investigación.

Entre el 2009 y el 2010, excepto Cuenca, ningún municipio lo hizo; doce $(60 \%)$ lo hicieron en el 2011; cuatro (20\%) en el 2012; uno (5\%) en el 2013; y dos (10\%) en el 2014. Resulta significativo que de los 26 municipios de este estudio, seis $(23,08 \%)$ no lo hayan hecho, siendo este un porcentaje muy alto.

Vemos entonces, que, ningún municipio cumple totalmente con todas las categorías de análisis. Solo uno cumple totalmente con cuatro de las cinco categorías. Luego, se observan varios cumplimientos parciales e incumplimientos.

DISCUSIÓN DE RESULTADOS

La investigación realizada, además de la descripción de lo encontrado en el análisis de las Ordenanzas seleccionadas, permite establecer que la normativa jurídica elaborada por los municipios para implementar la Silla Vacía, presenta serios problemas de elaboración en su estructura, de redacción y sobre todo (lo que resulta más importante) de inobservancia de las normas constitucionales y legales.
Una Ordenanza, es un conjunto de normas jurídicas de legislación de los municipios, dictada por el Concejo Cantonal que es precisamente su órgano legislativo. Debe contemplar necesariamente lo establecido en la Constitución y las leyes secundarias, en este caso concreto, la Ley Orgánica de Participación Ciudadana y el COOTAD. Además, debe presentar una estructura lógica, coherente y de aplicación práctica que recoja el espíritu, objetivo y propósito de lo que se quiere normar; situación ausente, en la gran mayoría de las ordenanzas analizadas.

El estudio de las ordenanzas y su confrontación con la Constitución y las leyes secundarias y el propósito conceptual y teórico de su existencia, constituye el basamento de la recomendación propuesta: un modelo de ordenanza que cumple con los objetivos señalados.

Los resultados obtenidos en la investigación realizada muestran que la demanda de que el Ecuador cuente con una democracia participativa, se ve dificultada por la falta de compromiso de las autoridades llamadas a posibilitarlo, incluso por mandato constitucional y legal, entre otras razones.

Esto se evidencia, en que no han gestionado o conseguido que se aprueben las normas jurídicas idóneas que permitan la participación ciudadana a través de la Silla Vacía. De esta forma, se impide que existan las condiciones mínimas que garanticen ese empoderamiento ciudadano en los términos que plantea Pachano (2011), o esa recuperación del espacio público, antes descrita.

Con la información proporcionada por la investigación realizada, podemos delimitar la necesidad de construir en primer lugar, normas jurídicas idóneas, constitucionales y legales que allanen el camino de la participación ciudadana, para luego pensar en posibilitarla de manera efectiva. En ese sentido, planteamos un proyecto de Ordenanza que cuente con el marco jurídico adecuado para estos fines.

\section{CONCLUSIONES}

En lo más relevante, se establece una constante en el sentido del incumplimiento de lo establecido en la Constitución, la Ley de Participación Ciudadana y el COOTAD:

- La convocatoria a las sesiones de los Concejos Cantonales no es lo suficientemente abierta, ni difundida entre toda la población. Por lo tanto, no es democrática. 
- Se condiciona, en muchos casos, que la participación ciudadana sea solo si se pertenece a una organización social; imposibilitando así que lo haga un ciudadano de manera individual o de mutuo propio.

- Establecen varios requisitos, algunos de ellos restrictivos, que limitan y condicionan la participación ciudadana.

- En algunos casos, se señala expresamente que determinados temas no serán susceptibles de ser tratados por la ciudadanía; estableciendo con ello una evidente inconstitucionalidad e ilegalidad.

- Salvo excepciones, no se establece en las ordenanzas, sanciones para las autoridades que incumplan las disposiciones legales relativas al tema.

No existe por parte de los municipios, la voluntad política de viabilizar la participación ciudadana a través del mecanismo de la Silla Vacía. Algunos de ellos ni siquiera han aprobado la ordenanza, como es el caso de la capital Quito.

Los que sí lo han hecho, se han encargado de elaborar y aprobar las respectivas ordenanzas más por su obligación legal de hacerlo, que por voluntad propia, logrando normas que reflejan muchas cortapisas, obstáculos, condiciones, limitaciones y restricciones.

\section{REFERENCIAS BIBLIOGRÁFICAS}

Acosta, A. (2011). Una caja de Herramientas. El "acumulado histórico" y la democracia participativa diseñada por la Constitución de 2008. De la lucha colectiva a la garantía constitucional. En D. Cornejo Menacho (Ed.), La silla vacía: el nuevo escenario para el diálogo de la sociedad civil y el estado. Un diagnóstico, 21-38. Quito: ESQUEL.

Alberich, T.; Espadas, M. A. (2011). Asociacionismo, participación ciudadana y políticas locales: planteamiento teórico y una experiencia práctica en Jaén. Alternativas. Cuadernos de Trabajo Social, 18, 119-146.

Burneo, L. (2011). Construcción de la ciudadanía mediante el uso cotidiano del espacio público. Recuperado de http://tesis.pucp.edu.pe/repositorio/handle/123456789/1147.

Constitución de la República del Ecuador (2008). Registro Oficial No. 449, 20 de octubre de 2008. CÓDIGO ORGÁNICO DE ORGANIZACIÓN TERRITORIAL, AUTONOMÍA Y DESCENTRALIZACIÓN (COOTAD), Registro Oficial No. 303, 19 de octubre de 2010.

INEC (2010) Censo de Población y Vivienda, Instituto Nacional de Estadística y Censos.

LEY ORGÁNICA DE PARTICIPACIÓN CIUDADANA Y CONTROL SOCIAL, Registro Oficial Suplemento No. 175, 20 de abril de 2010.

Pachano, S. (2011). Entre la cooptación y el miedo. El nuevo escenario para el diálogo de la sociedad civil y el Estado. De la letra de la Constitución a los procesos reales. En D. Cornejo Menchano (Ed.), La silla vacía: el nuevo escenario para el diálogo de la sociedad civil y el estado. un diagnóstico. Quito: ESQUEL.

Ramírez, A. (2009). Democracia participativa. La experiencia española contemporánea. Recuperado de www.rua.es/dspace/bistream/10045/14215/1/Tesis_Ramirez.pdf

Romero, E.; y Loja, D. (2011). El Fortalecimiento de la organización barrial en Ecuador como elemento necesario para el avance del Desarrollo Social. El Caso Loja. Recuperado de http:// www.eumed.net/rev/cccss/13/rflr.html

SENPLADES. (2009). Plan Nacional para el Buen Vivir. 2009- 2013. 